\title{
MicroRNA-126 inhibits endothelial permeability and apoptosis in apolipoprotein E-knockout mice fed a high-fat diet
}

\author{
XIAO-WEN CHENG ${ }^{1,2^{*}}$, YU-FENG WAN ${ }^{3 *}$, QING ZHOU ${ }^{2}$, YUAN WANG $^{2}$ and HUA-QING ZHU ${ }^{2}$ \\ ${ }^{1}$ Department of Clinical Laboratory, The First Affiliated Hospital of Anhui Medical University; ${ }^{2}$ Laboratory of \\ Molecular Biology and Department of Biochemistry, Anhui Medical University, Hefei, Anhui 230032; ${ }^{3}$ Department \\ of Otolaryngology, The Affiliated Chaohu Hospital of Anhui Medical University, Hefei, Anhui 238001, P.R. China
}

Received April 15, 2016; Accepted March 21, 2017

DOI: $10.3892 / \mathrm{mmr} .2017 .6952$

\begin{abstract}
Endothelial dysfunction and apoptosis have key roles in the initiation and progression of atherosclerosis (AS). AS has been demonstrated to be associated with a high-fat diet, which may increase endothelial permeability and apoptosis; however, the exact mechanisms underlying the development of AS remain poorly understood. MicroRNAs (miRNAs) are vital for the regulation of cardiovascular disease, and dysregulated miRNAs have been implicated in AS. The present study investigated whether miRNA (miR)-126 regulates high-fat diet-induced endothelial permeability and apoptosis by targeting transforming growth factor $\beta$ (TGF $\beta$ ), a secreted protein that controls cellular proliferation and apoptosis. In the present study, apolipoprotein $\mathrm{E}(\mathrm{apoE})^{-/-}$ mice were fed a high-fat diet in order to establish a model of AS. Mice were subcutaneously injected with a miR-126 mimic, a miR-126 antagomir or control miRNA. Reverse transcription-quantitative polymerase chain reaction was used to assess miR-126 expression, and a fluorometric assay was used to evaluate caspase- 3 activity. The effects of miR-126 on the endothelial permeability of the aortic intima were also explored. Western blotting and immunohistochemical analysis were used to investigate the effects of miR-126 on B-cell lymphoma-2 (Bcl-2) and transforming growth factor (TGF) $\beta$ protein expression levels. Furthermore, a luciferase assay was performed to verify whether TGF $\beta$ may be a direct target gene of miR-126. In apolipoprotein E-knockout mice, a high-fat diet reduced miR-126 expression and induced apoptosis as determined by the upregulation of caspase- 3 activity. A miR-126
\end{abstract}

Correspondence to: Dr Hua-Qing Zhu or Dr Yuan Wang, Laboratory of Molecular Biology and Department of Biochemistry, Anhui Medical University, 81 Meishan Road, Hefei, Anhui 230032, P.R. China

E-mail: aydzhq@126.com

E-mail: wangyuan@ahmu.edu.cn

"Contributed equally

Key words: microRNA-126, permeability, apoptosis, transforming growth factor $\beta$, high-fat diet antagomir increased endothelial permeability and apoptosis in mice fed a high-fat diet. By contrast, an miR-126 mimic attenuated endothelial permeability and apoptosis. The reduction in miR-126 was associated with a reduction in protein expression levels of Bcl-2 and an increase of TGF $\beta$ in mice fed a high-fat diet. In addition, the present study demonstrated that miR-126 reduced TGF $\beta$ expression following binding to the 3'-untranslated region of TGF $\beta$ mRNA. The current study demonstrated a role for miR-126 in AS and identified TGF $\beta$ as a direct target of miR-126. Furthermore, the present study demonstrated that miR-126 contributed to endothelial permeability and apoptosis, and suggested that the downregulation of TGF $\beta$ may be involved in the molecular mechanisms underlying the actions of miR-126. miR-126 may therefore have potential as a novel therapeutic target for the treatment of AS.

\section{Introduction}

Atherosclerosis (AS) is characterised by autoimmune and immunological mechanisms leading to plaque formation (1-5). Endothelial dysfunction and apoptosis are often seen as the initiating factors (3-6). A high-fat diet promotes the development of AS by increasing endothelial permeability and apoptosis (7-10). The discovery of microRNAs (miRNAs) has provided a novel perspective for AS research. miRNAs are small, single-stranded, non-coding RNA molecules ( 22 nucleotides) that are encoded within the genome and derived from endogenous small hairpin precursors $(3,4,11)$. miRNAs are involved in the post-transcriptional regulation of gene expression via binding to the 3'-untranslated regions (3'-UTRs) of specific mRNAs, which subsequently inhibits the transcription or translation of the target mRNAs $(12,13)$. It has been reported that miRNAs have key roles in various physiological cellular activities, including development, growth, proliferation, metabolism, differentiation and apoptosis. The occurrence and development of numerous cardiovascular diseases, including AS, hypertension and cardiac fibrosis, is associated with aberrant expression of miRNAs (14-17).

miRNA (miR)-126 is currently the only known miRNA expressed specifically in endothelial cells and hematopoietic stem cells, and is closely associated with AS, coronary heart disease and other cardiovascular diseases (18-22). Our recent study demonstrated that miR-126 exerts anti-apoptotic 
effects in human umbilical vein endothelial cells (HUVECs) by inhibiting the expression of tumour necrosis factor receptor-associated factor 7 and the production of reactive oxygen species (23).

Transforming growth factor $\beta$ (TGF $\beta$ ) is a cytokine that has key roles in numerous biological processes, including inflammatory responses and apoptosis (24-26). Studies have indicated that TGF $\beta$ is closely associated with endothelial cells and the pathogenesis of AS (27-31). The effect of miR-126 on high-fat diet-induced endothelial permeability and apoptosis remains unclear. The results of the present study suggested that miR-126 inhibited endothelial permeability and apoptosis by inhibiting TGF $\beta$ expression via binding to the 3 '-UTR of TGF $\beta$ mRNA.

\section{Materials and methods}

Cell culture and reagents. Primary HUVECs were purchased from Lonza, Inc. (Allendale, NJ, USA). Sulfo-NHS-LC-biotin was purchased from Pierce; Thermo Fisher Scientific, Inc. (Waltham, MA, USA). miRNA oligonucleotides (miR-126 mimic, 5'-CAG UAC UUU UGU GUA CAA-3'; miR-126 antagomir, 5'-CGC AUU AUU ACU CAC GGU ACG A-3'; and negative control miRNA, 5'-UUC UCC GAA CGU GUC ACG UTT-3') and TransMessenger Transfection reagent were obtained from Qiagen China Co., Ltd. (Shanghai, China). Rhodamine600 (XRITC)-avidin was obtained from Pierce; Thermo Fisher Scientific, Inc. HUVECs were cultured in endothelial cell growth medium supplemented with $10 \%$ fetal bovine serum and $100 \mathrm{U} / \mathrm{ml}$ penicillin-streptomycin (Invitrogen; Thermo Fisher Scientific, Inc.), and maintained at $37^{\circ} \mathrm{C}$ in a $5 \% \mathrm{CO}_{2}$ atmosphere. The cells at passage 3-4 were used for experiments. All experiments were repeated at least three times.

Animal experimental procedures. All of the experiments performed in the present study were approved by the Ethics Committee of The First Affiliated Hospital of Anhui Medical University (Hefei, China). A total of 60 male apolipoprotein $\mathrm{E}(\mathrm{apoE})^{-/-}$mice (age, 4 weeks; weight, $22 \pm 5 \mathrm{~g}$ ) were obtained from the Institute of Basic Medical Sciences of Peking Union Medical College (Beijing, China). Mice were housed individually in screen-bottomed plastic cages in a temperature-controlled room $\left(25^{\circ} \mathrm{C}\right)$ under a 12 -h light/dark cycle with free access to food and water. Mice were divided into two groups: Standard diet $(\mathrm{n}=16)$ and standard diet plus $5 \%$ lard oil and $2 \%$ cholesterol $(\mathrm{n}=44)$; diets were maintained for 16 weeks. At 12 weeks, 8 control and 8 high-fat diet mice were euthanized for detection of miR-126 expression levels, and the remaining control and high-fat diet mice were randomly divided into the following three groups: Control miRNA oligonucleotides $(n=8 ; 4$ control mice and 4 high-fat diet mice); miR-126 antagomir oligonucleotides $(n=18$; 9 control mice and 9 high-fat diet mice); and miR-126 mimic oligonucleotides ( $\mathrm{n}=18 ; 9$ control mice and 9 high-fat diet mice). Control miRNA, or miR-126 antagomir or mimic was injected subcutaneously at a dose of $10 \mathrm{mg} / \mathrm{kg}$ twice in the first week, followed by once a week for 4 weeks. During the study period, 1 mouse died in the control miRNA oligonucleotide group, 2 died in the miR-126 antagomir oligonucleotide group and 2 died in the miR-126 mimic oligonucleotide group. At the end of the experiment, the aorta was removed from mice as previously described (8). Part of the aorta was embedded in optimal cutting temperature compound and snap frozen. The remaining aorta was stored at $-80^{\circ} \mathrm{C}$.

miR-126 expression assay. Total RNA was extracted from the aorta using TRIzol ${ }^{\circledR}$ reagent (Invitrogen; Thermo Fisher Scientific, Inc.). miR-126 expression was determined using a miRNA Plate Assay kit (cat no. MA-0101; Signosis, Inc., Santa Clara, CA, USA) and an oligo mix specific for miR-126 (cat no. MO-0040; Signosis, Inc.), according to the manufacturer's protocol. The RNA content was normalized to U6 small nuclear (sn)RNA. miR-126 expression was also determined using reverse transcription-quantitative polymerase chain reaction (RT-qPCR) with the following primers: miR-126, forward 5'-UCGUACCGUGAGUAAUAAUGCG-3', reverse 5'-CAUUAUUACUCACGGUACGAUU-3'; and U6 snRNA, forward 5'-CTCGCTTCGGCAGCACA-3' and reverse 5'-AACGCTTCACGAATTTGCGT-3'. Amplification conditions were as follows: Initial denaturation at $95^{\circ} \mathrm{C}$ for $10 \mathrm{~min}$, followed by 40 cycles at $95^{\circ} \mathrm{C}$ for $15 \mathrm{sec}$, and at $60^{\circ} \mathrm{C}$ for $60 \mathrm{sec}$. The reaction mixture contained the following: 2X Power SYBR Green Master Mix (Applied Biosystems; Thermo Fisher Scientific, Inc.), cDNA template, $10 \mathrm{pmol} / \mu \mathrm{l}$ of each primer and sterile $\mathrm{H}_{2} \mathrm{O}$. Relative gene expression was quantified according to the comparative Cq method (32) using GraphPad Prism software version 5.01 (GraphPad Software, Inc., La Jolla, CA, USA).

Permeability assay. The permeability assay was performed as previously described (33). Briefly, frozen aorta sections $\left(10-\mu \mathrm{m}\right.$ thick) were stained at $37^{\circ} \mathrm{C}$ with Sulfo-NHS-LC-biotin for $30 \mathrm{~min}$, and blocked with $5 \%$ non-fat milk at $4^{\circ} \mathrm{C}$ overnight and subsequently submerged into blocking buffer containing XRITC-avidin (1:500) at room temperature for $1 \mathrm{~h}$. The slides were washed three times with PBS and dried. Images were captured using an Olympus Provis AX70 fluorescence microscope (Olympus Corporation, Tokyo, Japan).

Caspase-3 activity measurement. Caspase-3 activity was detected in aortic samples using a Caspase-3 Fluorometric assay kit (Enzo Life Sciences, Inc., Farmingdale, NY, USA), as previously described (34). Briefly, aortic samples were isolated from anesthetized mice and stored in liquid nitrogen. Samples were homogenized with lysis buffer containing $50 \mathrm{mM}$ Tris- $\mathrm{HCl}(\mathrm{pH}$ 6.8), $10 \%$ glycerol and 2\% SDS for $3 \mathrm{~min}$ on ice, maintained on ice for $10 \mathrm{~min}$, centrifuged at $21,130 \mathrm{x} \mathrm{g}$ for $10 \mathrm{~min}$ at $4^{\circ} \mathrm{C}$, and supernatants were collected and cryopreserved at $-70^{\circ} \mathrm{C}$ until further use. Protein concentration was determined using a bicinchoninic acid (BCA) assay with the Micro $\mathrm{BCA}^{\mathrm{TM}}$ Protein Assay kit (Pierce; Thermo Fisher Scientific, Inc.). Equal amounts of extracted protein samples $(50 \mu \mathrm{g})$ were incubated at $37^{\circ} \mathrm{C}$ overnight with $\mathrm{N}$-acetyl-Asp-Glu-Val-Asp p-nitroanilide (Ac-DEVD-pNA). The quantity of pNA that was released was estimated at $405 \mathrm{~nm}$ using a microplate ELISA reader. Caspase-3 relative activity was calculated as follows: Caspase-3 activity $=$ (mean experimental absorbance/mean control absorbance) $\mathrm{x} 100 \%$. 
Western blot analysis. Aortic samples were homogenized, lysed for $3 \mathrm{~min}$ on ice in 1X SDS lysis buffer containing $50 \mathrm{mM}$ Tris- $\mathrm{HCl}$ (pH 6.8), 10\% glycerol and 2\% SDS and boiled for $10 \mathrm{~min}$, followed by centrifugation at $16,000 \mathrm{x} \mathrm{g}$ for $10 \mathrm{~min}$ at room temperature. Protein concentration was determined using a Micro BCA ${ }^{\mathrm{TM}}$ Protein Assay kit (Pierce; Thermo Fisher Scientific, Inc.). Equal amounts of extracted protein samples $(20 \mu \mathrm{g})$ were separated by $10 \%$ SDS-PAGE and transferred onto polyvinylidene difluoride membranes (GE Healthcare Life Sciences, Chalfont, UK). Membranes were blocked using 5\% (w/v) bovine serum albumin (BSA; Amresco, LLC, Solon, $\mathrm{OH}, \mathrm{USA}$ ) for $2 \mathrm{~h}$ at room temperature, followed by incubation with the following primary antibodies: Anti-TGF $\beta$ (cat no. 3711; 1:1,000), anti-B-cell lymphoma (Bcl)-2 (cat no. $2872 ; 1: 1,000)$, purchased from Cell Signaling Technology, Inc. (Danvers, MA, USA); and anti-GAPDH (cat no. TA505454; 1:5,000; Beijing Zhongshan Jinqiao Biotechnology Co., Ltd., Beijing, China) diluted in TBS containing 0.05\% Tween-20 (TBST) at $4^{\circ} \mathrm{C}$ overnight. Following 3 washes with TBST, horseradish peroxidase (HRP)-conjugated secondary antibodies (cat nos. ZB-2305 and ZB-2301; 1:5,000; Beijing Zhongshan Jinqiao Biotechnology Co., Ltd.) were added to membranes and incubated at room temperature for $2 \mathrm{~h}$. Protein bands were visualized by enhanced chemiluminescence using ECL reagents (Pierce; Thermo Fisher Scientific, Inc.). GAPDH was used as the loading control. Blots were semi-quantified by densitometric analysis using the Image-Pro Plus software version 6.0 (Media Cybernetics, Inc., Rockville, MD, USA). Experiments were repeated 3 times.

Immunohistochemistry. Immunohistochemistry was performed as previously described (8). Sections $(10 \mu \mathrm{m})$ of the frozen aorta tissue samples were blocked using PBS containing $0.05 \%$ Tween-20 and $1 \%$ BSA at room temperature for $30 \mathrm{~min}$, and incubated at $4^{\circ} \mathrm{C}$ overnight with an anti-TGF $\beta$ (cat no. 3711; 1:1,000) primary antibody, purchased from Cell Signaling Technology, Inc. Subsequently, sections were incubated with a HRP-conjugated goat secondary antibody (cat no. ZB-2301; 1:5,000; Beijing Zhongshan Jinqiao Biotechnology Co., Ltd.) at room temperature for $60 \mathrm{~min}$. A Metal Enhanced 3,3'-diaminobenzidine Substrate kit (Pierce; Thermo Fisher Scientific, Inc.) was used for $3 \mathrm{~min}$ to develop the colour, and sections were counterstained with hematoxylin $(10 \%)$ at room temperature for $30 \mathrm{sec}$. The integral absorbance was examined under a light microscope and results were quantified using the Image-Pro Plus software version 6.0 (Media Cybernetics, Inc.).

Luciferase assay. The region of the TGF $\beta$ 3'-UTR containing the potential binding site of miR-126 (source: NCBI GenBank; https://www.ncbi.nlm.nih.gov/nuccore/NM_011577.2) was predicted using TargetScan version 7.1 (http://www.targetscan .org/vert_71/). The sequence was inserted into the 3 ' region of the luciferase gene in a luciferase vector (wt-Luc-TGF $\beta$; Shanghai GeneChem Co., Ltd., Shanghai, China), and a mutated version of this sequence was inserted into the vector (mu-Luc-TGF $\beta$ ). Plasmid DNA (300 ng) and miR-126 mimic, antagomir or control oligonucleotide $(80 \mathrm{nmol} / \mathrm{l})$ were co-transfected using TransMessenger Transfection reagent into HUVECs seeded into 6-well plates at a density of
$1-2 \times 10^{5}$ cells/well (confluence, $60-70 \%$ ) for $24 \mathrm{~h}$ at $37^{\circ} \mathrm{C}$. The pRL-TK vector (Promega Corporation, Madison, WI, USA) expressing Renilla luciferase served as a control. The luciferase assay was performed using a Dual-Luciferase ${ }^{\circledR}$ Reporter assay system (Promega Corporation) $24 \mathrm{~h}$ following transfection, according to the manufacturer's protocol.

Statistical analysis. Data are presented as the mean \pm standard deviation of 3 independent experiments. The statistical significance of the differences between groups was assessed using one-way analysis of variance followed by a Neuman-Keuls post hoc test for multiple comparisons. Comparisons between two groups were based on least significant differences. $\mathrm{P}<0.05$ was considered to indicate a statistically significant difference. Statistical analysis was performed using SPSS software version 21.0 (IBM Corp., Armonk, NY, USA).

\section{Results}

Reduction of miR-126 expression and increase in apoptosis of arterial endothelial cells in high-fat diet fed mice. To evaluate the impact of miR-126 on an established AS model, apoE $^{-/-}$mice were fed a high-fat diet for 12 weeks, followed by subcutaneous injection of $10 \mathrm{mg} / \mathrm{kg}$ control miRNA, miR-126 antagomir or miR-126 mimic twice for one week, and once a week for 4 weeks. Subsequently, the expression of miR-126 in the aorta was determined using a miRNA Plate assay kit. miR-126 levels were reduced in AS mice compared with control mice $(\mathrm{P}<0.05$; Fig. 1A). Following 4 weeks of treatment, the miR-126 mimic significantly increased miR-126 expression and the miR-126 antagomir significantly reduced the expression of miR-126 compared with AS mice injected with control miRNA ( $\mathrm{P}<0.05$; Fig. 1B). In addition, caspase-3 activity was measured following 4 weeks of treatment with the miRNAs. Compared with the control group, caspase-3 activity was greater in AS mice $(\mathrm{P}<0.05$; Fig. $1 \mathrm{C})$. Treatment with miR-126 antagomir and mimic exhibited no effect on caspase-3 activity in mice fed a standard diet (Fig. 1D). Thus, the standard diet group was not analysed in subsequent experiments.

miR-126 expression alleviates endothelial permeability in AS model mice. To investigate the effect of miR-126 on endothelial permeability, NHS-LC-biotin and XRITC-avidin were used to detect the endothelial permeability of aortic intima. Results presented in Fig. 2 demonstrated that the aortic intima from AS mice exhibited greater staining with NHS-LC-biotin compared with control mice, in which only the endothelial surface was stained. When AS mice were treated with an miR-126 antagomir (Fig. 2Ac), an increased quantity of NHS-LC-biotin leaked into the aortic intima compared with AS mice treated with control miRNA (Fig. 2Ab). By contrast, miR-126 mimic (Fig. 2Bc) treatment reduced endothelial permeability compared with control miRNA-treated AS mice (Fig. 2Bb).

miR-126 inhibits apoptosis in AS model mice. As apoptosis is involved in the development of AS, the effect of miR-126 on apoptosis was investigated in the current study. Caspase-3 activity was significantly increased in AS model mice treated with control miRNA compared with control mice treated with 
A

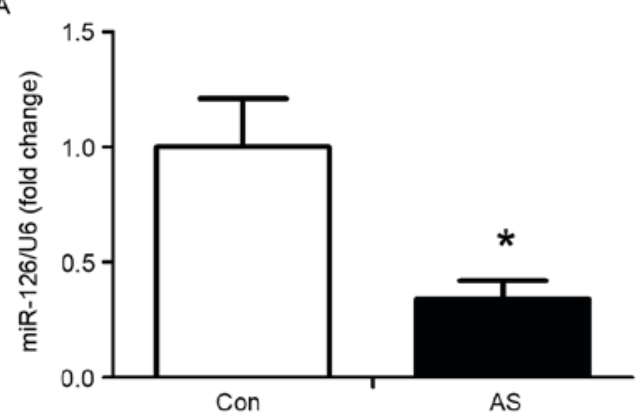

C

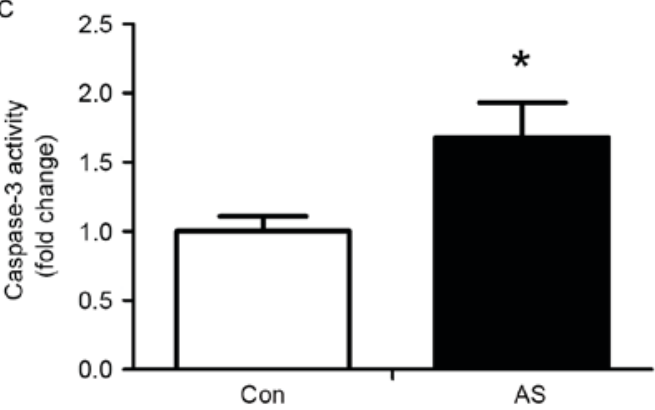

B
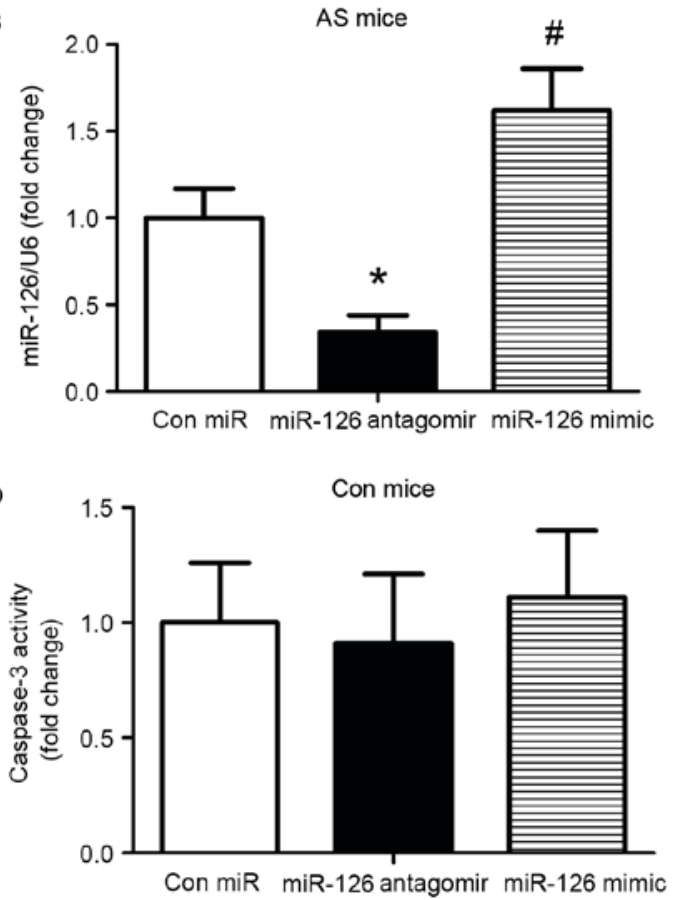

Figure 1. miR-126 expression and apoptosis in a mouse model of AS. AS was induced in mice by a high-fat diet; mice on a standard diet served as controls. (A) miR-126 expression levels in control and AS mice. (B) miR-126 expression was detected in AS mice treated with control miR, miR-126 antagomir or miR-126 mimic. Caspase-3 activity was measured in (C) control and AS mice and (D) control mice treated with control miR, miR-126 antagomir or miR-126 mimic. ${ }^{*} \mathrm{P}<0.05$ vs. control; ${ }^{\#} \mathrm{P}<0.05$ vs. miR-126 antagomir. miR, microRNA; AS, atherosclerosis; Con, control.

A
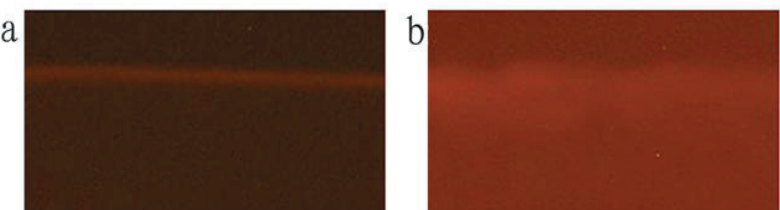

B
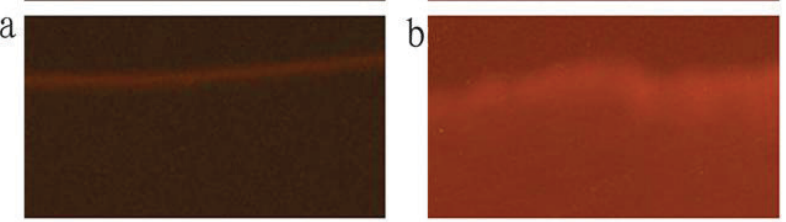

C

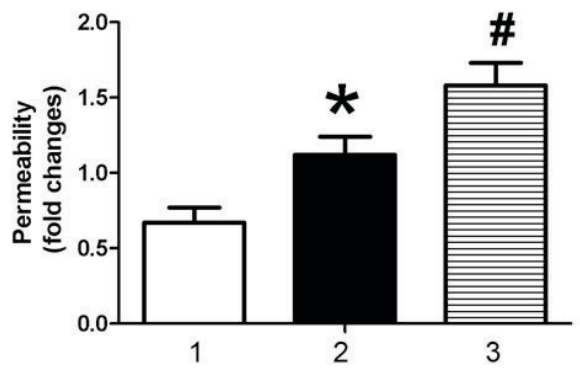

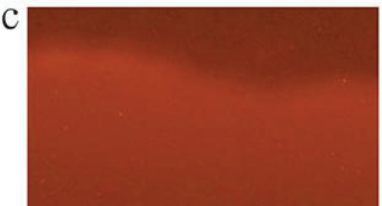
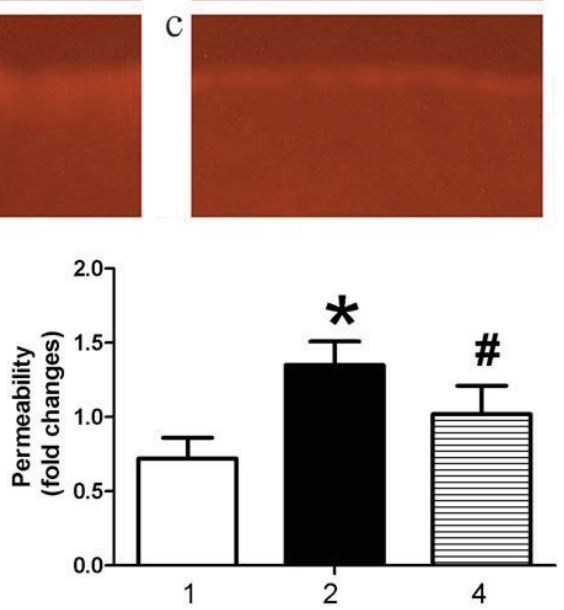

Figure 2. miR-126 expression levels affect endothelial permeability in the aorta. Mice were treated with (A) miR-126 antagomir or (B) miR-126 mimic. (a) Control mice treated with control miR, (b) AS mice treated with control miR and (c) AS mice treated with miR-126 antagomir (A)/mimic (B). (C) Following incubation of aortic intima sections with NHS-LC-biotin for $30 \mathrm{~min}$, Rhodamine600-avidin was used to detect surface-bound biotin. Magnification, x200. 1, control mice treated with control miR; 2, AS mice treated with control miR; 3, AS mice treated with miR-126 antagomir; and 4, AS mice treated with miR-126 mimic. ${ }^{*} \mathrm{P}<0.05$ vs. control mice treated with control miR; ${ }^{*} \mathrm{P}<0.05$ vs. AS mice treated with control miR. miR, microRNA; AS, atherosclerosis.

control miRNA, and miR-126 antagomir treatment further increased caspase-3 activity $(\mathrm{P}<0.05$; Fig. 3A). Treatment with an miR-126 mimic partially reversed the increased activity of caspase- 3 induced by a high-fat diet $(\mathrm{P}<0.05$; Fig. 3B). The opposite results were observed when the expression levels of the anti-apoptotic protein B-cell lymphoma 2 (Bcl-2) were analysed (Fig. 3C and D).

miR-126 modulates TGF $\beta$ expression in AS model mice. Previous studies have demonstrated that TGF $\beta$ promotes 
A

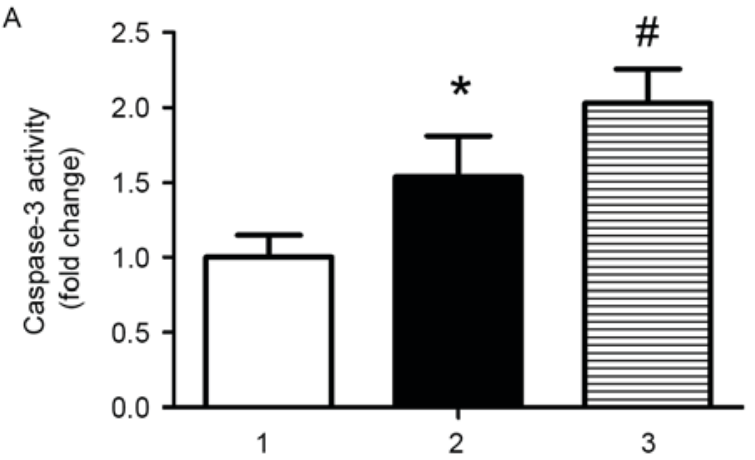

C
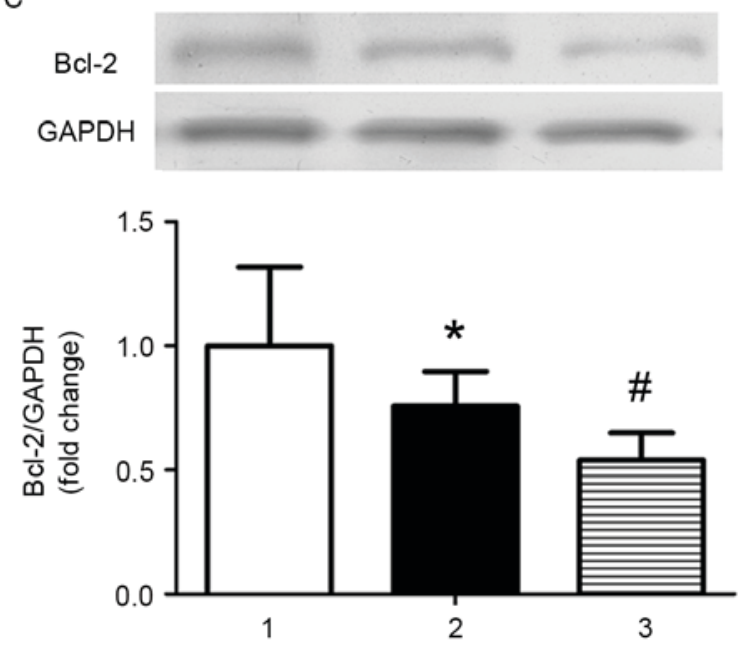

B

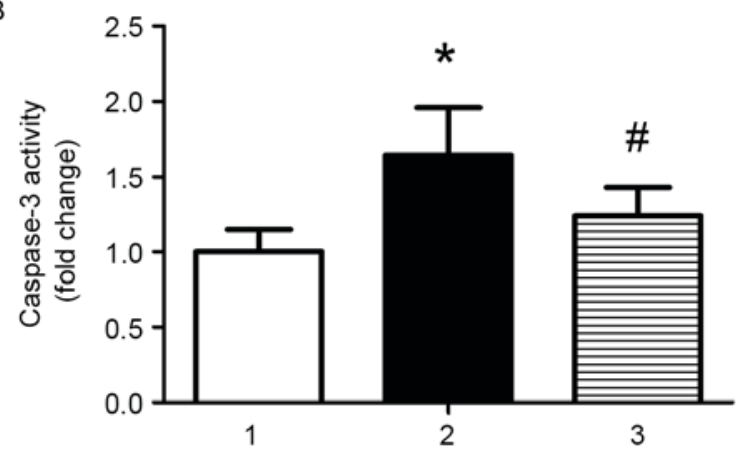

D
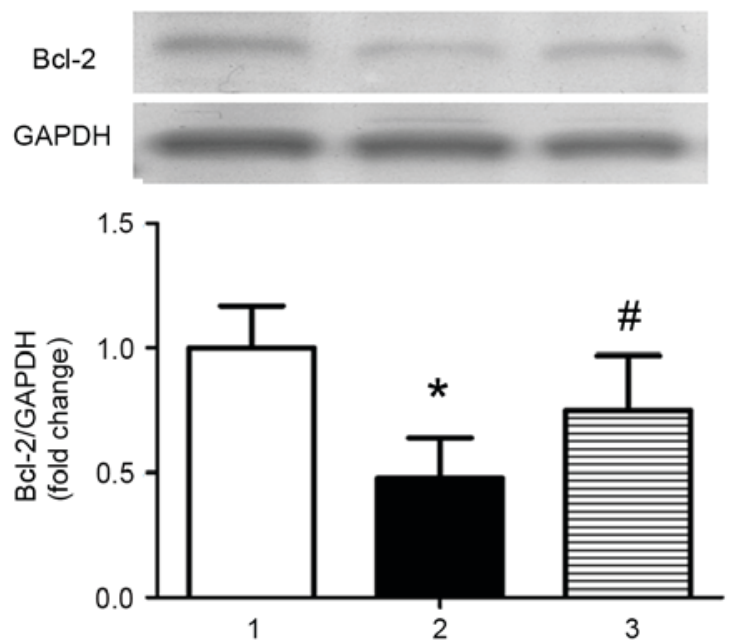

Figure 3. miR-126 expression affects caspase-3 activity and Bcl-2 protein expression levels in the aorta. The effect of (A) miR-126 antagomir and (B) miR-126 mimic on caspase-3 activity was measured using a Caspase-3 Fluorometric assay kit. Western blotting was performed to investigate the effect of (C) miR-126 antagomir and (D) miR-126 mimic on Bcl-2 protein expression levels in the aorta of AS mice. 1, control mice treated with control miR; 2, AS mice treated with control miR; and 3, AS mice treated with miR-126 antagomir (A and C)/mimic (B and D). ${ }^{*} \mathrm{P}<0.05$ vs. control mice treated with control miR; ${ }^{*} \mathrm{P}<0.05$ vs. AS mice treated with control miR. miR, microRNA; AS, atherosclerosis; Bcl-2, B-cell lymphoma 2.

apoptosis in endothelial cells (22-24). Therefore, the present study investigated the effect of miR-126 on the expression of TGF $\beta$. TGF $\beta$ protein expression levels were increased in AS model mice treated with control miRNA compared with control mice treated with control miRNA, as determined by western blot analysis (Fig. 4A and B) and immunohistochemistry (Fig. 4C and D). This result indicated that TGF $\beta$ may be a target gene of miR-126. Results in Fig. 4 demonstrated that miR-126 antagomir treatment significantly increased levels of TGF $\beta$ in AS model mice (Fig. 4A and C), whereas an miR-126 mimic had the opposite effect (Fig. 4B and D).

$T G F \beta$ is a target of $m i R-126$. miRNAs specifically bind to the 3'-UTR of target mRNAs and induce transcript degradation or translational repression. An miR-126 binding site in the TGF $\beta$ 3'-UTR was identified and the alignment between miR-126 and the wild-type and mutant TGF $\beta$ 3'-UTR are presented in Fig. 5A and B, respectively. This indicated that miR-126 may cause translation inhibition of TGF $\beta$. To validate this result of bioinformatics analysis, wt-Luc-TGF $\beta$ and mu-Luc-TGF $\beta$ were inserted into the luciferase vector, which was co-transfected into HUVECs with control miRNA, miR-126 antagomir or miR-126 mimic. Treatment with miR-126 mimic reduced luciferase activity in HUVECs co-transfected with wt-Luc-TGF $\beta$ compared with HUVECs co-transfected with wt-Luc-TGF $\beta$ and control miRNA (Fig. 5C). In HUVECs co-transfected with mu-Luc-TGF $\beta$ and an miR-126 mimic, no inhibition was observed (Fig. 5C). miR-126 antagomir significantly promoted luciferase activity in the wt-Luc-TGF $\beta$-transfected HUVECs, but had no effect on mu-Luc-TGF $\beta$-transfected HUVECs (Fig. 5D).

\section{Discussion}

The present study investigated the effect of alterations in miR-126 expression on endothelial cell permeability and apoptosis in a mouse model of AS. The present results demonstrated that TGF $\beta$ was a direct target gene for miR-126, thus suggesting that miR-126 may bind to TGF $\beta$ and suppresses its expression. These findings indicated a novel mechanism by which miR-126 may have a vital role in endothelial permeability and apoptosis in AS model mice.

It has been reported that a high-fat diet is one of the factors that promotes AS development (7,31,35). Jakob et al (36) demonstrated that the expression of miR-126 in patients with chronic heart failure was significantly reduced compared with healthy controls. Furthermore, cardiac function was markedly improved by transfection with miR-126. The present study 
A
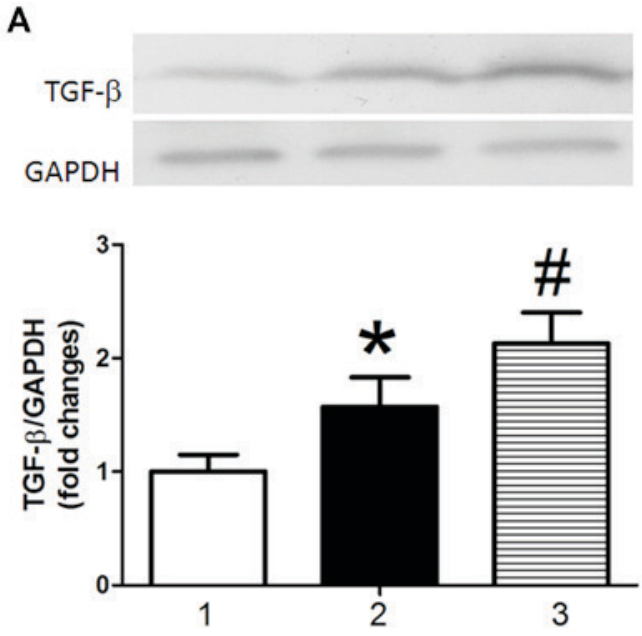

C

a

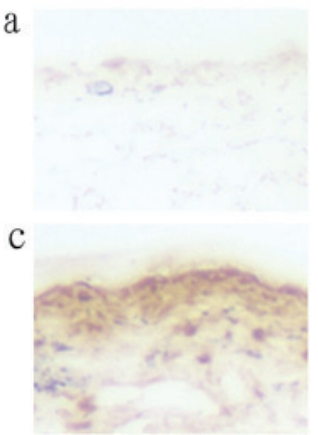

b
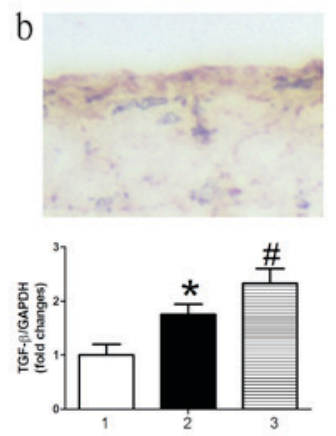

B TGF- $\beta$

GAPDH

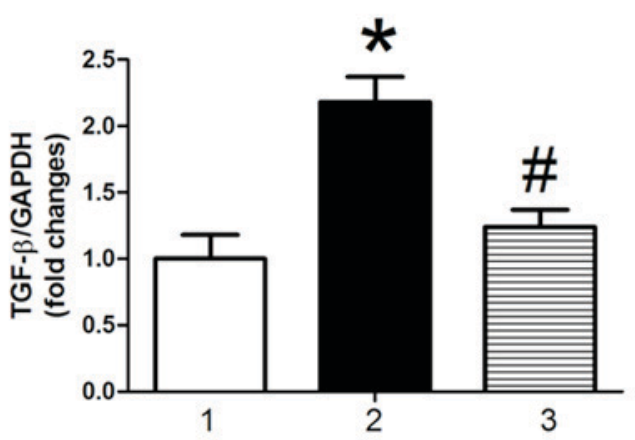

D

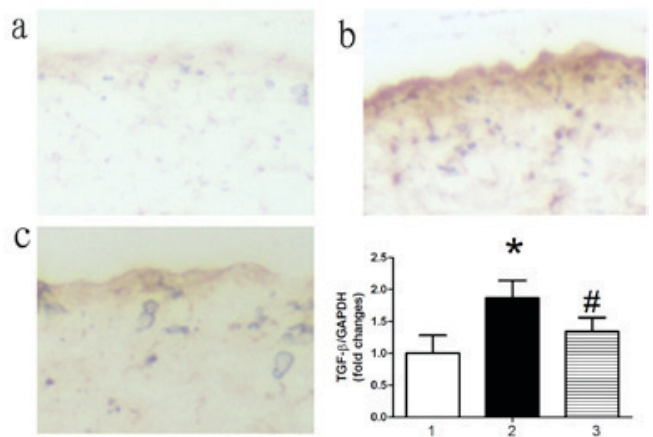

Figure 4. miR-126 expression affects TGF $\beta$ protein expression levels in the aorta. Western blotting was performed to investigate the effect of (A) miR-126 antagomir and (B) miR-126 mimic on TGF $\beta$ protein expression levels. Immunohistochemistry was performed to investigate the effect of (C) miR-126 antagomir and (D) miR-126 mimic on TGF $\beta$ protein expression. (a) Control mice treated with control miR, (b) AS mice treated with control miR and (c) AS mice treated with miR-126 antagomir (C)/mimic (D). Magnification x 200. 1, control mice treated with control miR; 2, AS mice treated with control miR; and 3, AS mice treated with miR-126 antagomir (A and C)/mimic (B and D). ${ }^{\mathrm{P}}<0.05$ vs. control mice treated with control miR; ${ }^{\mathrm{P}}<0.05$ vs. AS mice treated with control miR. miR, microRNA; TGF $\beta$, transforming growth factor $\beta$; AS, atherosclerosis.

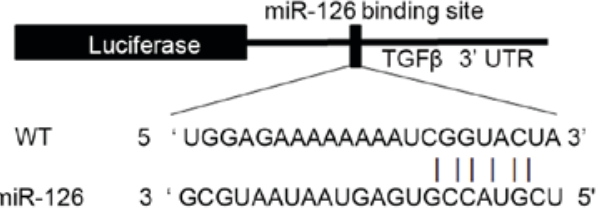

B

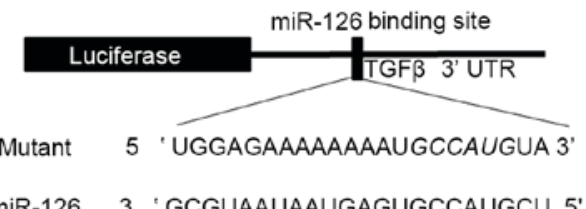

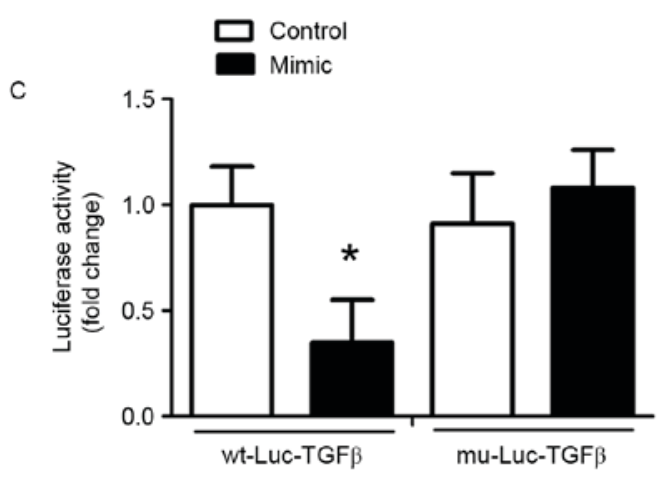

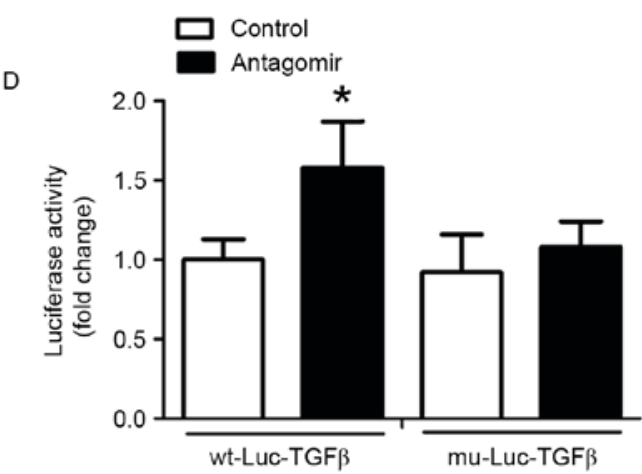

Figure 5. miR-126 binds to and inhibits TGF $\beta$ expression in HUVECs. A Dual-Luciferase assay was performed. (A) A segment of the TGF $\beta$ 3'UTR containing the predicted miR-126 binding site was inserted downstream of the luciferase-coding sequence. Sequence alignment demonstrates TGF $\beta$ 3'UTR complementarity with the 5 ' end of miR-126. (B) Sequence alignment of miR-126 and the mutated TGF $\beta$ 3'UTR exhibited no complementarity. (C) HUVECs were co-transfected with the wt-Luc-TGF- $\beta$ or mu-Luc-TGF $\beta$ plasmid and miR-126 mimic or control miR. (D) HUVECs were co-transfected with the wt-Luc-TGF $\beta$ or mu-Luc-TGF $\beta$ plasmid and miR-126 antagomir or a control miR. "P<0.05 vs. control. miR, microRNA; TGF $\beta$, transforming growth factor $\beta$; HUVECs, human umbilical vein endothelial cells; UTR, untranslated region; wt, wild-type; mu, mutant; Luc, luciferase. 
demonstrated that arterial wall permeability and apoptosis was increased in AS, and inhibition of miR-126 promoted this pathological process. Overexpression of miR-126, using an miR-126 mimic, attenuated these effects. Thus, normal levels of miR-126 may be necessary for the integrity of the arterial wall. However, miR-126 inhibited apoptosis as demonstrated by reduced caspase- 3 activity and increased protein expression levels of Bcl-2 in mice fed high-fat diets. miR-126 had no effect on basal apoptosis in mice fed standard chow. This indicated that miR-126 may only inhibit apoptosis in mice on a high-fat diet, which inhibits miR-126 expression. However, this requires further investigation.

Typically, miRNAs influence gene expression by inducing post-transcriptional inhibition, mRNA degradation or translation suppression $(12,13)$. It has been reported that TGF $\beta$ has key roles in inflammatory responses and apoptosis (24-26), and is closely associated with the pathogenesis of $\operatorname{AS}(27,28)$. The present study demonstrated that miR-126 directly binds to TGF $\beta$ mRNA and inhibits its expression. The results of the current study demonstrated that a reduction in miR-126 expression caused by a high-fat diet increased TGF $\beta$ protein expression levels and caused an increase in endothelial permeability and apoptosis.

In conclusion, the present study identified TGF $\beta$ as a direct target gene of miR-126, and demonstrated that reduced miR-126 expression increased TGF $\beta$ protein expression. These findings suggested that TGF $\beta$ downregulation may be implicated in the increased endothelial leakage and apoptosis that is observed in AS. Based on the results of the current study, miR-126 may have potential as a novel target for the treatment of cardiovascular diseases.

\section{Acknowledgements}

The present study was supported by the National Natural Science Foundation of China (grant nos. 81570419, 81470568 and 81270372), the National Natural Science Fund for Distinguished Young Scholars of China (grant no. 81302150) and Grants for Cultivating Program of National Natural Science Foundation for Young Scholars of the First Affiliated Hospital of Anhui Medical University (grant no. 2012KJ10).

\section{References}

1. Staff AC, Johnsen GM, Dechend R and Redman CW Preeclampsia and uteroplacental acute atherosis: Immune and inflammatory factors. J Reprod Immunol 101: 120-126, 2014.

2. Ooi CY, Sutcliffe MP, Davenport AP and Maguire JJ: Changes in biomechanical properties of the coronary artery wall contribute to maintained contractile responses to endothelin-1 in atherosclerosis. Life Sci 118: 424-429, 2014.

3. Schober A, Nazari-Jahantigh $M$ and Weber C: MicroRNA-mediated mechanisms of the cellular stress response in atherosclerosis. Nat Rev Cardiol 12: 361-174, 2015.

4. Svoboda P: A toolbox for miRNA analysis. FEBS Lett 589: 1694-1701, 2015.

5. Xu S, Liu Z and Liu P: Targeting hydrogen sulfide as a promising therapeutic strategy for atherosclerosis. Int J Cardiol 172 : 313-317, 2014.

6. Lloyd MM, Grima MA, Rayner BS, Hadfield KA, Davies MJ and Hawkins CL: Comparative reactivity of the myeloperoxidase-derived oxidants hypochlorous acid and hypothiocyanous acid with humancoronary artery endothelial cells. Free Radic Biol Med 65: 1352-1362, 2013
7. Nahrendorf M and Swirski FK: Lifestyle effects on hematopoiesis and atherosclerosis. Circ Res 116: 884-894, 2015.

8. Zhu HQ, li Q, Dong LY, Zhou Q, Wang H and Wang Y: MicroRNA-29b promotes high-fat diet-stimulated endothelial permeability and apoptosis in apoE knock-out mice by down-regulating MT-1 expression. Int J Cardiol 176: 764-770, 2014.

9. Kraakman MJ, Kammoun HL, Allen TL, Deswaerte V, Henstridge DC, Estevez E, Matthews VB, Neill B, White DA, Murphy AJ, et al: Blocking IL-6 trans-signaling prevents high-fat diet-induced adipose tissue macrophage recruitment but does not improve insulin resistance. Cell Metab 21: 403-416, 2015.

10. van Bussel BC, Henry RM, Ferreira I, van Greevenbroek MM, van der Kallen CJ, Twisk JW, Feskens EJ, Schalkwijk CG and Stehouwer CD: A healthy diet is associated with less endothelial dysfunction and less low-grade inflammation over a 7-year period in adults at risk of cardiovascular disease. J Nutr 145: 532-540, 2015.

11. Romaine SP, Tomaszewski M, Condorelli G and Samani NJ: MicroRNAs in cardiovascular disease: An introduction for clinicians. Heart 101: 921-928, 2015.

12. Andreou I, Sun X, Stone PH, Edelman ER and Feinberg MW: miRNAs in atherosclerotic plaque initiation, progression, and rupture. Trends Mol Med 21: 307-318, 2015.

13. Wang W, Zhang E and Lin C: MicroRNAs in tumor angiogenesis. Life Sci 136: 28-35, 2015.

14. Thum $\mathrm{T}$ and Condorelli G: Long noncoding RNAs and microRNAs in cardiovascular pathophysiology. Circ Res 116: 751-762, 2015.

15. Loyer X, Mallat Z, Boulanger CM and Tedgui A: MicroRNAs as therapeutic targets in atherosclerosis. Expert Opin Ther Targets 19: 489-496, 2015.

16. Nazari-Jahantigh M, Egea V, Schober A and Weber C: MicroRNA-specific regulatory mechanisms in atherosclerosis. J Mol Cell Cardiol 89: 35-41, 2015.

17. Orenes-Piñero E, Montoro-García S, Patel JV, Valdés M, Marín F and Lip GY: Role of microRNAs in cardiac remodelling: New insights and future perspectives. Int J Cardiol 167: 1651-1659, 2013.

18. Fish JE, Santoro MM, Morton SU, Yu S, Yeh RF, Wythe JD, Ivey KN, Bruneau BG, Stainier DY and Srivastava D: microRNA-126 regulates angiogenic signaling and vascular integrity. Dev Cell 15: 272-284, 2008.

19. Synetos A, Toutouzas K, Stathogiannis K, Latsios G, Tsiamis E, Tousoulis D and Stefanadis C: microRNAs in arterial hypertension. Curr Top Med Chem 13: 1527-1532, 2013.

20. Hulsmans M and Holvoet P: MicroRNA-containing microvesicles regulating inflammation in association with atherosclerotic disease. Cardiovasc Res 100: 7-18, 2013.

21. Sun X, Zhang M, Sanagawa A, Mori C, Ito S, Iwaki S, Satoh H and Fujii S: Circulating microRNA-126 in patients with coronary artery disease: Correlation with LDL cholesterol. Thromb J 10: 16,2012 .

22. Long G, Wang F, Duan Q, Chen F, Yang S, Gong W, Wang Y, Chen $C$ and Wang DW: Human circulating microRNA-1 and microRNA-126 as potential novel indicators for acute myocardial infarction. Int J Biol Sci 8: 811-818, 2012.

23. Wang Y, Wang F, Wu Y, Zuo L, Zhang S, Zhou Q, Wei W, Wang Y and Zhu H: MicroRNA-126 attenuates palmitate-induced apoptosis by targeting TRAF7 in HUVECs. Mol Cell Biochem 399: 123-130, 2015.

24. Franken R, den Hartog AW, de Waard V, Engele L, Radonic T, Lutter R, Timmermans J, Scholte AJ, van den Berg MP, Zwinderman $\mathrm{AH}$, et al: Circulating transforming growth factor- $\beta$ as a prognostic biomarker in Marfan syndrome. Int J Cardiol 168: 2441-2446, 2013.

25. Yan F, Wang Y, Wu X, Peshavariya HM, Dusting GJ, Zhang M and Jiang F: Nox4 and redox signaling mediate TGF- $\beta$-induced endothelial cell apoptosis and phenotypic switch. Cell Death Dis 5: e1010, 2014.

26. Frei K, Gramatzki D, Tritschler I, Schroeder JJ, Espinoza L, Rushing EJ and Weller M: Transforming growth factor- $\beta$ pathway activity in glioblastoma. Oncotarget 6: 5963-5977, 2015

27. Rath D, Chatterjee M, Müller I, Müller K, Böckmann C, Droppa M, Stimpfle F, Karathanos A, Borst O, Seizer P, et al: Platelet expression of transforming growth factor beta 1 is enhanced and associated with cardiovascular prognosis in patients with acute coronary syndrome. Atherosclerosis 237: 754-759, 2014. 
28. Hwang JS, Eun SY, Ham SA, Yoo T, Lee WJ, Paek KS, Do JT, Lim DS and Seo HG: PPARס modulates oxLDL-induced apoptosis of vascular smooth muscle cells through a TGF- $\beta /$ FAK signaling axis. Int J Biochem Cell Biol 62: 54-61, 2015.

29. Tian H, Liu J, Chen J, Gatza ML and Blobe GC: Fibulin-3 is a novel TGF- $\beta$ pathway inhibitor in the breast cancer microenvironment. Oncogene 34: 5635-5647, 2015.

30. Marcantoni E, Dovizio M, O Gaora P, Di Francesco L, Bendaya I, Schiavone S, Trenti A, Guillem-Llobat P, Zambon A, Nardelli GB, et al: Dysregulation of gene expression in human fetal endothelial cells from gestational diabetes in response to TGF- $\beta 1$. Prostaglandins Other Lipid Mediat 120: 103-114, 2015.

31. Climent M, Quintavalle M, Miragoli M, Chen J, Condorelli G and Elia L: TG-F $\beta$ triggers miR-143/145 transfer from smooth muscle cells to endothelial cells, thereby modulating vessel stabilization. Circ Res 116: 1753-1764, 2015.

32. Livak KJ and Schmittgen TD: Analysis of relative gene expression data using real-time quantitative PCR and the 2 (-Delta Delta C(T)) method. Methods 25: 402-408, 2001.
33. Zhu HQ, Zhou Q, Jiang ZK, Gui SY and Wang Y: Association of aorta intima permeability with myosin light chain kinase expression in hypercholesterolemic rabbits. Mol Cell Biochem 347: 209-215, 2011.

34. Zhu H, Yang Y, Wang Y, Li J, Schiller PW and Peng T: MicroRNA-195 promotes palmitate-induced apoptosis in cardiomyocytes by down-regulating Sirt1. Cardiovasc Res 92: 75-84, 2011.

35. Hu ZP, Fang XL, Fang N, Wang XB, Qian HY, Cao Z, Cheng Y, Wang BN and Wang Y: Melatonin ameliorates vascular endothelial dysfunction, inflammation, and atherosclerosis by suppressing the TLR4/NF- $\kappa \mathrm{B}$ system in high-fat-fed rabbits. J Pineal Res 55: 388-398, 2013.

36. Jakob P, Doerries C, Briand S, Mocharla P, Kränkel N, Besler C, Mueller M, Manes C, Templin C, Baltes C, et al: Loss of angiomiR-126 and 130a in angiogenic early outgrowth cells from patients with chronic heart failure: Role for impaired in vivo neovascularization and cardiac repair capacity. Circulation 126 : 2962-2975, 2012. 